\title{
PREFACE TO THE SPECIAL SECTION ON ALGORITHMS AND ARCHITECTURES FOR REAL-TIME CONTROL
}

\author{
A.E.B. Ruano* and P.J. Fleming * * \\ *Unidade de Ciencias Exactas e Humanas, University of Algarve, Campus de Gambelas, 8000 Faro, Partugal \\ (aruano@mozart.si.ualg.pt) \\ **Department of Automatic Control and Systems Engineering, University of Sheffield, Mappin Street, Sheffield S1 3JD, UK
}

(Received May 1997; in final form August 1997)

Rapid developments in microelectronics and computer science continue to fuel opportunities for real-time control engineers. These openings arise from drivers such as ever-increasing system complexity and sophistication, environmental legislation, economic competition, safety and reliability. These are typical of the themes that were highlighted at the IFAC Workshop on Algorithms and Architectures for Real-Time Control (AARTC '97).

The Portuguese Society for Automatic Control (APCA) hosted AARTC '97, which was held at Vilamoura, Portugal. This Workshop was the fourth in the series. Previous Workshops have been held at Bangor, UK (September 1991), Seoul, Korea (August/September 1992), and Ostend, Belgium (May/June 1995). In a new development for this Workshop series, the International Program Committee required submission of full draft papers for selection. As a result, it subsequently proved difficult to select the 85 papers which constituted the Final Programme from the original 115 high-quality contributions received. The final programme consisted of 22 sessions covering major areas of software, hardware and applications for real-time control. Important topics were scheduling, "soft" computing methods, software tools and architectures, embedded systems, parallel and distributed systems, architectures, custom processors, algorithms, estimation methods, neural networks, fuzzy methods, PID controllers, transport applications, robotics and discrete-event and hybrid systems.

It is in the nature of IFAC Workshops that a proportion of papers will describe emerging research, which requires further work before being eligible for journal publication. However, the following six papers were selected by the IPC as representative and mature examples of the research described at the meeting.

The first two papers address hardware issues in parallel and distributed computing. Industry-based authors, Darbyshire and Kerry, describe a large highperformance multiple-processor system for active control and general signal processing. One application, described here, employs active control to reduce vibration in a complex mechanical structure, a magnetically levitated ship's machinery raft, having 32 three-axis electromagnetic actuators and over 500 sensors. The second paper (Pontremoli and Pereira) identifies the need for dedicated hardware units for task scheduling and communication in distributed real-time systems. The success of such systems relies on timely execution of technical tasks. Their solution, based on low-cost microcontrollers, is designed to free up more processing time for application tasks, thereby enhancing predictability of performance.

The second pair of papers concerns robotic applications. Pashkevich ingeniously proposes practical and reliable inverse kinematics methods which, unlike many suggested solutions, do not use Jacobians but rather are based on one-dimensional iterative searches that incorporate kinematic subroutines available in most commercial robot controllers. These real-time algorithms have been embedded in real-time software of commercial controllers and applied to welding-cell design in an automotive plant. Batista, Peixoto, and Araújo combine parallel computing with a paradigm known as active/purposive vision to implement a real-time 
control architecture for complex robotics systems. Rather than passively acquiring image information, their vision system interacts with the environment, acquiring relevant image information for its purpose by using agents that work in a co-operative manner.

The last pair of papers describe practical schemes for real-time process control and estimation. With examples drawn from the water utility industry, Cox, Daniel and Lowdon demonstrate an effective scheme for determining PI and PPI (predictive PI) controller parameters. Of course, their method has wider applicability in process control. This paper has a strong applications emphasis; for example, showing the advantages of pPI control in certain circumstances while hailing its acceptability by process operators.
The paper by Cunha, Couto, and Ruano rounds off the set appropriately with an environmental control problem common to climates such as those found in Portugal. Greenhouse air temperature model parameters are estimated in real-time with the twin goals of tighter temperature regulation and energy efficiency. Practical experiments demonstrate the efficacy of their recursive identification method.

Following the undoubted success of AARTC 97 in Portugal, the 5th IFAC AARTC Workshop is planned for Cancun, Mexico on 15-17 April 1998 and details of this event may be found at http://www.shef.ac.uk/ acse/aartc/. Finally, the editors wish to express their deep appreciation of the strenuous efforts of IPC members, and their assistance in the selection of papers for this Special Section. 